Journal of Universal Mathematics

Vol.4 No.2 PP.166-171 (2021)

ISSN-2618-5660

DOI: $10.33773 /$ jum. 964785

\title{
NEW TYPES OF SETS IN ČECH CLOSURE SPACES
}

\author{
NAİME DEMIRTAŞ AND ORHAN DALKILIÇ
}

0000-0003-4137-4810 and 0000-0003-3875-1398

\begin{abstract}
In this paper, we analysis and introduce the concepts of regular closed (open) sets and regular generalized closed (open) sets in Čech closure spaces. Also, we investigate the properties such as intersection, union, subspaces of regular generalized closed (open) sets of a Čech closure spaces. Moreover, by giving counter examples of one-sided theorems, it has been shown that the converse situation is not realized.
\end{abstract}

\section{INTRODUCTION}

In a topological space, many types of sets such as open set, closed set, generalized closed set, generalized open set, regular generalized closed set, regular open, regular closed are defined. Firstly, studies in this area started with the generalized closed set model that Levine [8] put forward by generalizing closed sets of any topological space. For example, it was shown that completeness, normality, compactness in a uniform space are inherited by generalized closed subsets. Balachandran et al. [9] introduced the concept of generalized continuous maps by using generalized closed sets. In the following years, Palaniappan and Chandrasekhara Rao [10] introduced regular generalized closed sets in topological spaces.

There are many methods researchers can use to define a topology. Cech closure space, one of these methods, are a set of axioms used to define a topology on a set other than any null set by E. Čech in [3]. After defining Čech closure spaces, it has managed to attract the attention of many researchers and then studied on these spaces, see e.g. $[4,5,6,7]$.

Thanks to this paper, regular generalized closed (open) sets and regular closed (open) sets, which are two new concepts for Čech closure spaces, are brought into literature. Moreover, the given sets were analyzed in detail and their properties such as subspaces, intersection, union were examined. In addition, the types of sets such as closed sets, generalized closed sets given previously for Čech closure spaces

Date: Received: 2021-07-07; Accepted: 2021-07-26 .

2000 Mathematics Subject Classification. 03E72; 54E99.

Key words and phrases. Regular Closed Set, Regular Generalized Closed Set, Čech Closure Space. 
and the new set types given in this paper were compared and the relationships between each other were studied.

\section{PRELIMINARIES}

In this section, we recall some basic notions in Čech closure spaces.

Throughout this paper, let $\mathcal{U} \neq \emptyset$ be a set, $2^{\mathcal{U}}$ denotes the power set of $\mathcal{U}$ and $X, Y$ be non-empty subsets of $U$.

Definition 2.1. [1] An operator $c: 2^{\mathcal{U}} \rightarrow 2^{\mathcal{U}}$ defined satisfying the axioms:

$[c 1] c(\emptyset)=\emptyset$,

$[c 2] X \subseteq c(X)$ for all $X \subseteq \mathcal{U}$,

[c3] $c(X \cup Y)=c(X) \cup c(Y)$ for all $X, Y \subseteq \mathcal{U}$

is called a Cech closure operator (briefly closure operator) and the pair $(\mathcal{U}, c)$ is called a Čech closure space (briefly closure space). Here, for $X \subset \mathcal{U}$, we call $c(X)$ the closure of $X$.

Definition 2.2. [1] Let $c$ be a closure operator and $(\mathcal{U}, c)$ be a closure space. Then, for $\emptyset \neq X \subseteq \mathcal{U}$,

(i) A $c$ on $\mathcal{U}$ is called idempotent if $c(X)=c(c(X))$.

(ii) $X$ is closed set (briefly c-set) in $(\mathcal{U}, c)$ if $X=c(X)$.

(iii) $X$ is open set (briefly o-set) in $(\mathcal{U}, c)$ if its complement is c-set.

(iv) The $\emptyset$ and $\mathcal{U}$ are both o-set and c-set.

Definition 2.3. [1] Let $(\mathcal{U}, c)$ be a closure space. A closure space $\left(\mathcal{V}, c_{\mathcal{V}}\right)$ is called a subspace of $(\mathcal{U}, c)$ if $\mathcal{V} \subseteq \mathcal{U}$ and $c_{\mathcal{V}}(X)=c(X) \cap \mathcal{V}$, for all $X \subseteq \mathcal{V}$.

Definition 2.4. [1] Let $\left(\mathcal{V}, c_{\mathcal{V}}\right)$ be a Cech closure subspace of $(\mathcal{U}, c)$. If $K$ is a closed subset of $\left(\mathcal{V}, c_{\mathcal{V}}\right)$, then $K$ is a closed subset of $(\mathcal{U}, c)$.

Definition 2.5. [2] Let $(\mathcal{U}, c)$ be a closure space. Then,

(i) A $X \subseteq \mathcal{U}$ is called a generalized closed set (briefly gc-set), if $c(X) \subseteq K$ whenever $K$ is an open subset of $(\mathcal{U}, c)$ with $X \subseteq Y$.

(ii) A $X \subseteq \mathcal{U}$ is called a generalized open set (briefly go-set), if its complement is gc-set.

(iii) If $X$ and $Y$ are generalized closed subsets of $(\mathcal{U}, c)$, then $X \cup Y$ is gc-set. Moreover, the $X \cap Y$ need not be a gc-set.

Remark 2.6. [2] Every c-set is gc-set. The converse need not be a c-set.

Definition 2.7. [1] An interior operator on $\mathcal{U}$ is a map int $: 2^{\mathcal{U}} \rightarrow 2^{\mathcal{U}}$ which satisfies

(i) $\operatorname{int}(\mathcal{U})=\mathcal{U}$,

(ii) $\operatorname{int}(X) \subseteq X$ for all $X \subseteq \mathcal{U}$,

(iii) $\operatorname{int}(X \cap Y)=\operatorname{int}(X) \cap \operatorname{int}(Y)$ for all $X, Y \subseteq \mathcal{U}$.

In other words, the set $\operatorname{int}(X)$ with respect to the closure operator $c$ is defined as $\operatorname{int}(X)=\mathcal{U}-c(\mathcal{U}-X)$. 


\section{REGULER GENERALIZED CLOSED SETS}

Definition 3.1. Let $(\mathcal{U}, c)$ be a closure space. A $X \subseteq \mathcal{U}$ is called a regular closed set (briefly rc-set) [regular open set (briefly ro-set)], if $X=c(\operatorname{int}(X))[X=\operatorname{int}(c(X))]$.

Remark 3.2. Every ro-set is o-set. The converse need not be a ro-set as can be seen from the following example.

Example 3.3. Let $\mathcal{U}=\{m, n\}$ and define a closure operator $c$ on $\mathcal{U}$ by $c(\emptyset)=\emptyset$ and $c(\{m\})=c(\{n\})=c(\mathcal{U})=\mathcal{U}$. Then $\{m\}$ and $\{n\}$ are o-sets but they are not ro-sets.

Definition 3.4. Let $(\mathcal{U}, c)$ be a closure space. A $X \subseteq \mathcal{U}$ is called a regular generalized closed set (briefly rgc-set) iff $c(X) \subseteq K$ whenever $X \subseteq K$, where $K$ is ro-set.

Proposition 1. Let $(\mathcal{U}, c)$ be a closure space. If $X$ and $Y$ are regular generalized closed subsets of $(\mathcal{U}, c)$, then $X \cup Y$ is rgc-set.

Proof. Let $K$ be a regular open subset of $(\mathcal{U}, c)$ such that $X \cup Y \subseteq K$. Then $X \subseteq K$ and $Y \subseteq K$. Since $X$ and $Y$ are rgc-set, $c(X) \subseteq K$ and $c(Y) \subseteq K$. Therefore $c(X) \cup c(Y) \subseteq K$ and hence $c(X \cup Y) \subseteq K$. Consequently $X \cup Y$ is rgc-set.

Remark 3.5. The intersection of two rgc-sets is generally not a rgc set.

Example 3.6. Let $\mathcal{U}=\{m, n, r\}$ and define a closure operator $c$ on $\mathcal{U}$ by $c(\emptyset)=\emptyset$, $c(\{m\})=\{m, n\}, c(\{n\})=c(\{r\})=c(\{n, r\})=\{n, r\}, c(\{m, n\})=c(\{m, r\})=$ $c(\mathcal{U})=\mathcal{U}$. Then $\{m, n\}$ and $\{m, r\}$ are rgc-set but $\{m, n\} \cap\{m, r\}=\{m\}$ is not rgc-set.

Proposition 2. Let $(\mathcal{U}, c)$ be a closure space. If $X$ is a rgc-set and $K$ is a c-set in $(\mathcal{U}, c)$, then $X \cap K$ is rgc-set.

Proof. Let $G$ be an regular open subset of $(\mathcal{U}, c)$ such that $X \cap K \subseteq G$. Then $X \subseteq G \cup(\mathcal{U}-K)$ and so $c(X) \subseteq G \cup(\mathcal{U}-K)$. Therefore $c(X) \cap K \subseteq G$. Since $K$ is a c-set, $c(X \cap K) \subseteq G$. Hence, $X \cap K$ is a rgc-set.

Proposition 3. Let $\left(\mathcal{V}, c_{\mathcal{V}}\right)$ be a closed subspace of $(\mathcal{U}, c)$. If $K$ is a regular generalized closed subset of $(\mathcal{V}, c \mathcal{V})$, then $K$ is a regular generalized closed subset of $(\mathcal{U}, c)$.

Proof. Let $G$ be an regular open subset of $(\mathcal{U}, c)$ such that $K \subseteq G$. Then $K \subseteq G \cap \mathcal{V}$. Since $K$ is a rgc-set and $G \cap \mathcal{V}$ is a ro-set in $\left(\mathcal{V}, c_{\mathcal{V}}\right), c(K) \cap \mathcal{V}=c_{\mathcal{V}}(K) \subseteq G$. But $\mathcal{V}$ is a closed subset of $(\mathcal{U}, c)$ and $c(K) \subseteq G$. Hence, $K$ is a regular generalized closed subset of $(\mathcal{U}, c)$.

Theorem 3.7. Let $(\mathcal{U}, c)$ be a closure space and $c$ be idempotent. If $X$ is a regular generalized closed subset of $(\mathcal{U}, c)$ and $X \subseteq Y \subseteq c(X)$, then $c(Y)-Y$ contains no nonempty rc-set.

Proof. Since $Y \subseteq c(X)$ and $c$ is idempotent, then $c(Y) \subseteq c(c(X))=c(X)$. That is $c(Y) \subseteq c(X)$. Since $X \subseteq Y$, we obtain $\mathcal{U}-Y \subseteq \mathcal{U}-X$. Form $c(Y) \subseteq c(X)$ and $\mathcal{U}-Y \subseteq \mathcal{U}-X,(u(Y) \cap(\mathcal{U}-Y)) \subseteq(c(X) \cap(\mathcal{U}-X))$ which implies $(c(Y)-Y) \subseteq$ $(c(X)-X)$. Now $X$ is a rgc-set. Hence $c(X)-X$ has no nonempty regular generalized closed subset, neither does $c(Y)-Y$. 
Theorem 3.8. Let $(\mathcal{U}, c)$ be a closure space and $X \subseteq \mathcal{U}$. If $X$ is a rgc-set, then $c(X)-X$ contains no nonempty rc-set.

Proof. Suppose that $X$ is a rgc-set. Let $Y$ be a regular generalized closed subset of $c(X)-X$. Then $Y \subseteq c(X) \cap(\mathcal{U}-X)$ and so $X \subseteq \mathcal{U}-Y$. But $X$ is a rgcset. Therefore $c(X) \subseteq \overline{\mathcal{U}}-Y$. Consequently $Y \subseteq \mathcal{U}-c(X)$. Since $Y \subseteq c(X)$, $Y \subseteq c(X) \cap(\mathcal{U}-c(X))=\emptyset$. Thus $B=\emptyset$. Therefore $c(X)-X$ contains no nonempty rc-set.

The converse of this theorem is not true in general as can be seen from the following example.

Example 3.9. Let $\mathcal{U}=\{x, y, z\}$ and define a closure operator $c$ on $\mathcal{U}$ by $c(\emptyset)=\emptyset$, $c(\{x\})=\{x, y\}, c(\{y\})=c(\{z\})=c(\{y, z\})=\{y, z\}, c(\{x, y\})=c(\{x, z\})=$ $c(\mathcal{U})=\mathcal{U}$. Then $c(\{x\})-\{x\}=\{y\}$ does not contain nonempty rc-set. But $\{x\}$ is not rgc-set.

Corollary 1. Let $(\mathcal{U}, c)$ be a closure space and $X$ be a rgc-subset of $(\mathcal{U}, c)$. Then $X$ is a rc-set if and only if $c(\operatorname{int}(X))-X$ is a rc-set.

Proof. Let $X$ be regular generalized closed subset of $(\mathcal{U}, c)$. If $X$ is a rc-set, then $c(\operatorname{int}(X))-X=\emptyset$. But $\emptyset$ is always a rc-set. Therefore $c(\operatorname{int}(X))-X$ is a rc-set.

Conversely, suppose that $c(\operatorname{int}(X))-X$ is a rc-set. But $X$ is a rgc-set. Also $c(X)-X$ contains the rc-set $c(\operatorname{int}(X))-X$. By Theorem 3.8, we have $c(\operatorname{int}(X))-$ $X=\emptyset$. Hence $c(\operatorname{int}(X))=X$. Therefore $X$ is a rc-set.

Theorem 3.10. Let $(\mathcal{U}, c)$ be a closure space and $X \subseteq \mathcal{U}$. If $X$ is a gc-set, then $X$ is a rgc-set.

Proof. Suppose that $X \subseteq K$, where $K$ is a ro-set. Now $K$ is a ro-set, implies that $K$ is a open. Since $X$ is a gc-set, then $c(X) \subseteq K$. Therefore $X$ is a rgc-set.

The converse of this theorem is not true in general as can be seen from the following example.

Example 3.11. Let $\mathcal{U}=\{1,2,3,4\}$ and define a closure operator $c$ on $\mathcal{U}$ by

$$
\begin{gathered}
c(\emptyset)=\emptyset, \quad c(\{1\})=c(\{1,2\})=\{1,2\}, \quad c(\{2\})=\{2\}, \\
c(\{3\})=c(\{2,3\})=\{2,3\}, \quad c(\{4\})=\{4\}, \quad c(\{2,4\})=\{2,4\}, \\
c(\{1,3\})=c(\{1,2,3\})=\{1,2,3\}, \quad c(\{1,4\})=c(\{1,2,4\})=\{1,2,4\}, \\
c(\{3,4\})=c(\{2,3,4\})=\{2,3,4\}, \quad c(\{1,3,4\})=c(\mathcal{U})=\mathcal{U} .
\end{gathered}
$$

Then $\{1,3\}$ is a rgc-set but it is not gc-set.

Definition 3.12. Let $(\mathcal{U}, c)$ be a closure space. A $X \subseteq \mathcal{U}$ is called a regular generalized open set (briefly a rgo-set) if and only if its complement is a rgc-set.

Theorem 3.13. Let $(\mathcal{U}, c)$ be a closure space. A set $X \subseteq \mathcal{U}$ is a rgo-set if and only if $H \subseteq \operatorname{int}(X)$ whenever $H$ is a rc-set and $H \subseteq X$.

Proof. Let $H \subseteq \operatorname{int}(X)$ whenever $H$ is a rc-set, $H \subseteq X$ and $K=\mathcal{U}-X$. Suppose that $K \subseteq G$ where $G$ is a ro-set.

Now $T \subseteq G$ implies $H=\mathcal{U}-G \subseteq X$ and $H$ is a rc-set which implies $H \subseteq$ $\operatorname{int}(X)$. Also $H \subseteq \operatorname{int}(X)$ implies $\mathcal{U}-\operatorname{int}(X) \subseteq \mathcal{U}-H=G$. This inturn implies $\mathcal{U}-\operatorname{int}(\mathcal{U}-K) \subseteq G$. Or equivalently $c(K) \subseteq G$. Thus $K$ is a rgc-set. Hence we obtain $X$ is a a rgo-set. 
Conversely, suppose that $X$ is a rgo-set, $H \subseteq X$ and $H$ is a rc-set. Moreover $\mathcal{U}-H$ is a ro-set. Then $\mathcal{U}-X \subseteq \mathcal{U}-H$. Since $\mathcal{U}-X$ is a $\operatorname{rgc}$-set, $\mathcal{U}-c(X) \subseteq \mathcal{U}-H$. Therefore $H \subseteq \mathcal{U}-(\mathcal{U}-c(X))=\operatorname{int}(X)$.

Theorem 3.14. Let $(\mathcal{U}, c)$ be a closure space. If $X$ is a rgo-subset of $(\mathcal{U}, c)$, then $G=\mathcal{U}$ whenever $G$ is a ro-set and $\operatorname{int}(X) \cup(\mathcal{U}-X) \subseteq G$.

Proof. Suppose that $X$ is a rgo-set in $(\mathcal{U}, c)$. Let $G$ be a ro-set and $\operatorname{int}(X) \cup$ $(\mathcal{U}-X) \subseteq G$. This implies $\mathcal{U}-G \subseteq(\mathcal{U}-\operatorname{int}(X)) \cap(\mathcal{U}-(\mathcal{U}-X))$. That is $\mathcal{U}-G \subseteq(\mathcal{U}-\operatorname{int}(X)) \cap X$ or equivalently $\mathcal{U}-G \subseteq(\mathcal{U}-\operatorname{int}(X))-(\mathcal{U}-X)=$ $(\mathcal{U}-(\mathcal{U}-c(\mathcal{U}-X)))-(\mathcal{U}-X)=c(\mathcal{U}-X)-(\mathcal{U}-X)=c(\mathcal{U}-X)-(\mathcal{U}-X)$. Now $\mathcal{U}-G$ is a rc-set and $\mathcal{U}-X$ is rgc-set. By Theorem 3.8, it follows taht $\mathcal{U}-G=\emptyset$. Hence we obtain $G=\mathcal{U}$.

Theorem 3.15. Let $(\mathcal{U}, c)$ be a closure space. If $X$ is a rgc-subset of $(\mathcal{U}, c)$, then $c(X)-X$ is a rgo-set.

Proof. Suppose that $X$ is a rgc-set and $H \subseteq c(X)-X$, where $H$ is a rc-set. By Theorem 3.8, $H=\emptyset$ and so $H \subseteq \operatorname{int}(c(X)-X)$. By Theorem 3.13, $c(X)-X$ is a rgo-set.

The converse of this theorem is not true in general as can be seen from the following example.

Example 3.16. Let $\mathcal{U}=\{m, n, r, s\}$ and define a closure operator $c$ on $\mathcal{U}$ by

$$
\begin{gathered}
c(\emptyset)=\emptyset, \quad c(\{m\})=c(\{m, n\})=\{m, n\}, \quad c(\{n\})=\{n\}, \\
c(\{r\})=c(\{n, r\})=\{n, r\}, \quad c(\{s\})=\{s\}, \quad c(\{n, s\})=\{n, s\}, \\
c(\{m, r\})=c(\{m, n, r\})=\{m, n, r\}, \quad c(\{m, s\})=c(\{m, n, s\})=\{m, n, s\}, \\
c(\{r, s\})=c(\{n, r, s\})=\{n, r, s\}, \quad c(\{m, r, s\})=c(\mathcal{U})=\mathcal{U} .
\end{gathered}
$$

Then $c(\{r\})-\{r\}=\{n\}$ is a rgo-set. But $\{r\}$ is not a rgc-set in $(\mathcal{U}, c)$.

\section{Conclusion}

In the present paper, we have introduced regular closed (open) sets and regular generalized closed (open) sets in Čech closure spaces. In addition, some basic properties of new concepts for Čech closure spaces were examined. We have investigated behavior relative to union, intersection, subspaces of regular closed (open) sets and regular generalized closed (open) sets. We hope that the findings in this paper will

help researcher enhance and promote the further study on Čech closure spaces to carry out a general framework.

\section{Acknowledgments}

The authors would like to thank the reviewers and editors of Journal of Universal Mathematics.

\section{Funding}

The author(s) declared that has no received any financial support for the research, authorship or publication of this study.

The Declaration of Conflict of Interest/ Common Interest

The author(s) declared that no conflict of interest or common interest 


\section{The Declaration of Ethics Committee Approval}

This study does not be necessary ethical committee permission or any special permission.

\section{The Declaration of Research and Publication Ethics}

The author(s) declared that they comply with the scientific, ethical, and citation rules of Journal of Universal Mathematics in all processes of the study and that they do not make any falsification on the data collected. Besides, the author(s) declared that Journal of Universal Mathematics and its editorial board have no responsibility for any ethical violations that may be encountered and this study has not been evaluated in any academic publication environment other than Journal of Universal Mathematics.

\section{REFERENCES}

[1] K. Chandrasekhara Rao, R. Gowri, On closure space, Varahmithir Journal of Mathematics Sciences, Vol.5, No.2, pp.375-378, (2005).

[2] C. Boonpok, Generalized closed sets in Čech closed spaces. Acta Universitatis Apulensis. Mathematics-Informatics, Vol.22, pp.133-140, (2010).

[3] E. Čech, Topological Spaces, Topological Papers of Eduard Čech, Academia, Preque, pp.436472, (1968).

[4] J. Chvalina, On homeomorphic topologies and equivalent set-systems, Arch. Math. 2, Scripta Fac. Sci. Nat. UJEP Brunensis, Vol.12, pp.107-116, (1976).

[5] J. Chvalina, Stackbases in power sets of neighbourhood spaces preserving the continuity of mappings, Arch. Math. 2, Scripta Fac. Sci. Nat. UJEP Brunensis, Vol.17, pp.81-86, (1981).

[6] L. Skula, Systeme von stetigen abbildungen, Czech. math. J., Vol. 17, No.92, 45-52, (1967).

[7] J. Šlapal, Closure operations for digital topology, Theoret. Comput. Sci., Vol.305, pp.457-471, (2003).

[8] N. Levine, Generalized closed sets in topology, Rend. Circ. Mat. Palermo, Vol.19, pp.89-96, (1970).

[9] K. Balachandran, P. Sundaram, H. Maki, On generalized continuous maps in topological spaces, Mem. Fac. Sci. Kochi Univ. Ser. A Math., Vol.12, pp.5-13, (1991).

[10] M. Palaniappan, K. Chandrasekhara Rao, Regular Generalized Closed Sets, Kyungpook Mathematical Journal, Vol.33, No.2, pp.211-219, (1993).

(Naime Demirtaş) Mersin University, Faculty of Science and Art, Department of Mathematics, Mersin, Turkey

Email address, Naime DEMIRTAŞ: naimedemirtas@mersin.edu.tr

(Orhan DALKiliÇ) Mersin University, Faculty of Science and Art, Department of Mathematics, Mersin, Turkey

Email address, Orhan DALKILIÇ: orhandlk952495@hotmail.com 